\title{
Audit on postanaesthetic patient handover at the recovery room, completing the audit cycle
}

\begin{abstract}
Background: Postoperative patient handover by anaesthetists to recovery room staff is a core part of postoperative care. This includes the transfer of information about the patients' preoperative condition, the nature of surgery and anaesthesia (including any intraoperative problems) and postoperative management plan. To improve patient handover in the recovery room, we choose to adopt "patient handover standards" from the Australian Medical Association and British Doctors Committee. These standards were introduced at an audit meeting. Following this introduction, we performed an audit which demonstrated poor compliance. A training program was instituted and re-audit demonstrated an improvement in adherence with the standards.
\end{abstract}

Methods: We performed an audit on postanaesthetic patient handover using the standards set out in " patient handover standards" from the Australian Medical Association, 2006 and British Doctors Committee, 2004. This was carried out in the recovery room in Gondar University hospital between March 18- May 27, 2013. A trained observer recorded the handover process against all the eleven elements of the standard. Information was collected from a total of 124 handovers taking place between 30 anaesthetists and 12 nurses in the recovery room. This included a wide range of surgical specialities, and both general and regional anaesthesia.

Results: The first audit result revealed that postoperative patient handover practice of anaesthetists was poor in the areas (percentages show level of correct performance based on the standards) of patient identity $3.2 \%$, preoperative patient condition $0 \%$, type of operation $82.2 \%$, type of anaesthesia $82.2 \%$, intraoperative vital signs $87.1 \%$, intraoperative analgesia use $62.9 \%$, intraoperative fluid management $59.7 \%$, intraoperative blood loss $8.1 \%$, intraoperative clinical incidents $3.2 \%$, recovery condition $45.1 \%$ and postoperative management plan $3.2 \%$. These areas of practice were improved after training.

Conclusion and recommendation: The postoperative patient handover practice of graduate anaesthetists (finished undergraduate course) was remarkably improved after training. We suggest that regular training on postoperative patient handover needs to be provided for both graduate and qualified anaesthetists. Moreover, regular re-auditing is required until the anaesthetists meet the standards and to ensure patient safety in the course of postoperative patient care.

Keywords: postoperative, patient handover, graduate anaesthetists, recovery room
Volume 8 Issue 2 - 2017

Endale Gebreegziabher Gebremedhn,' Nigussie Yigzaw Muluneh, ${ }^{2}$ Amare Demissie Ayele, ${ }^{3}$ Dereje Hailu Anbesse, ${ }^{4}$ Abebe Woldeselassie Tewolde, ${ }^{2}$ Endeshaw Admassu Cherkos,5 Kiber Temesgen Anteneh, ${ }^{5}$ Markos Tezera Taye, ${ }^{6}$ Tezera Gessesse Asfaw 'Department of Anaesthesia, School of Medicine, College of Medicine and Health Sciences, University of Gondar, Ethiopia ${ }^{2}$ Department of Psychiatry, School of Medicine, College of Medicine and Health Sciences, University of Gondar, Ethiopia ${ }^{3}$ Department of Nursing, College of Medicine and Health Sciences, University of Gondar, Ethiopia

${ }^{4}$ Department of Optometry, School of Medicine, College of Medicine and Health Sciences, University of Gondar, Ethiopia ${ }^{5}$ Department of Midwifery, College of Medicine and Health Sciences, University of Gondar, Ethiopia

${ }^{6}$ Department of Quality of Education, Higher Diploma Coordinator, University of Gondar, Ethiopia ${ }^{7}$ Department of English, Higher Diploma Coordinator, University of Gondar, Ethiopia

Correspondence: Endale Gebreegziabher Gebremedhn, Department of Anaesthesia, School of Medicine, College of Medicine and Health Sciences, University of Gondar, P.O.Box 196, Gondar, Ethiopia, Email endalege2I@gmail.com

Received: May 14, 2017 | Published: June 08, 2017

\section{Introduction}

Australian Medical Association defined patient handover as the transfer of professional responsibility and accountability for some or all aspects of care for a patient, or group of patients, to another person or professional group on a temporary or permanent basis. ${ }^{1}$ The aim of patient handover is to provide a high quality and appropriate clinical information to the coming healthcare professionals to allow for the safe transfer of responsibility for the care of patients. Good handovers are essential in providing the continuity of care, patient safety and error avoidance. This will help to ensure that after handover all members of the team will have the same understanding. Individual nurses and interns have a duty to accept responsibility for the assessment and continuing care of every patient coming from the operation theatre until they formally transfer the care of patients to other professionals. ${ }^{2-8}$ The major responsibility of the anaesthetist during anaesthesia is to provide a high quality care for the patients. This requires the continuous presence of the anaesthetist and close monitoring of the condition of the patients. Postoperative patient handover to the recovery room staffs is also one of the main parts of the anaesthetists' duty. This includes the transfer of information about preoperative and intraoperative conditions and postoperative management plans. ${ }^{910}$ Graduate anaesthetists (anaesthetist who finished undergraduate course) are expected to handover all the relevant information to the recovery room staff. The aim of this audit was to assess the level of postoperative patient handover practice of graduate anaesthetists and to improve the continuity and quality of post operative patient care.

\section{Materials and methods}

Permission was obtained from the institutional ethical review committee. We performed an audit on postanaesthetic patient handover using "patient handover standards" adopted from the Australian Medical Association, 2006 and British Doctors Committee, 2004 (Table 1). This was carried out in the recovery room in Gondar University hospital between March18- May 27, 2013.

A. Inclusion criteria: All (adult, paediatric, major-minor, elective) patients from different specialities: surgery, obstetrics and gynaecology underwent operation both under general anaesthesia and regional anaesthesia who were handovered during the study period were audited. However, patients undergoing emergency operation were excluded. 
B. Audit sample size and sampling method: The handovers of sixty two patients were audited during the first phase of an audit. The same numbers of patients were also handovered and audited during the second phase.

C. Data collector training: Two BSc holder nurses were trained about the standards by giving lecture for one day and role play for two days to overcome the interpersonal variation between the two data collectors. They used checklist during the actual observation in the recovery room. But the checklist was not attached with the patients' charts because of lack of budget to duplicate the checklist.

\section{Result}

Information was collected from a total of 124 handovers taking place between 30 anaesthetists and 12 nurses in the recovery room during the data collection time. This included a wide range of surgical specialities, and both general and regional anaesthesia.

\section{Results of the first audit}

Information was collected from 62 patients. The postoperative patient handover practice of anaesthetists was poor in the areas of patient identity $3.2 \%$, preoperative patient condition $0 \%$, type of operation $82.2 \%$, type of anaesthesia $82.2 \%$, intraoperative vital signs $87.1 \%$, intraoperative analgesia use $62.9 \%$, intraoperative fluid management $59.7 \%$, intraoperative blood loss $8.1 \%$, intraoperative clinical incidents $3.1 \%$, recovery condition $45.1 \%$ and postoperative management plan $3.2 \%$ (Table 1 ).

A. Action: These results were presented to the anaesthetic and recovery staff at an audit meeting. Following this, training was instituted in the use of the handover standards and the standards were posted in the operation theatres and in the recovery room. A re-audited was performed.

\section{Results of the second audit}

Information was collected from 62 patients. Patient identity $77.4 \%$, preoperative patient condition $82.2 \%$, type of operation $100 \%$, type of anaesthesia $100 \%$, intraoperative vital signs $95.1 \%$, intraoperative analgesia use $91.9 \%$, intraoperative fluid management $91.9 \%$, intraoperative blood loss $79.0 \%$, intraoperative clinical incidents reporting $45.1 \%$, recovery condition $95.1 \%$ and postoperative management plan $95.1 \%$ (Table 3 ).

Table I Standards for postoperative patient handover, the University of Gondar teaching hospital

\begin{tabular}{|c|c|c|c|c|c|}
\hline S. No. & Standards & Exceptions & Target & Source of evidence & Where the data will be found \\
\hline $\mathrm{I}$ & Patient identity explained & None & $100 \%$ & BMA \& AMA,NHS safe handover & Checklist \\
\hline 2 & Preoperative patient status discussed & None & $100 \%$ & BMA \& AMA,NHS safe handover & Checklist \\
\hline 3 & Type of operation explained & None & $100 \%$ & BMA \& AMA,NHS safe handover & Checklist \\
\hline 4 & Type of anaesthesia explained & None & $100 \%$ & BMA \& AMA,NHS safe handover & Checklist \\
\hline 5 & Intraoperative vital signs discussed & None & $100 \%$ & BMA \& AMA,NHS safe handover & Checklist \\
\hline 6 & Analgesic drugs given during intraoperatively explained & None & $100 \%$ & BMA \& AMA,NHS safe handover & Checklist \\
\hline 7 & Intraoperative fluid management discussed & None & $100 \%$ & BMA \& AMA,NHS safe handover & Checklist \\
\hline 8 & Intraoperative blood loss and transfusion explained & None & $100 \%$ & BMA \& AMA,NHS safe handover & Checklist \\
\hline 9 & Intraoperative clinical incident discussed & None & $100 \%$ & BMA \& AMA,NHS safe handover & Checklist \\
\hline 10 & Recover condition of the patients explained & None & $100 \%$ & BMA \& AMA,NHS safe handover & Checklist \\
\hline II & Postoperative management plan briefly discussed & None & $100 \%$ & BMA \& AMA,NHS safe handover & Checklist \\
\hline
\end{tabular}

BMA: British Medical Association;AMA:Australian Medical Association; NHS: National Health Safety

Table 2 Pre-training results of postoperative patient handover of patients at the recovery room, March I8- April I0, 20I3 (N=62)

\begin{tabular}{|c|c|c|c|c|}
\hline S. No. & Standard & Handover & $\begin{array}{l}\text { Total number of patients for } \\
\text { whom the standards were } \\
\text { addressed }\end{array}$ & $\begin{array}{l}\text { Total percentage } \\
(\%)\end{array}$ \\
\hline $\mathrm{I}$ & Patient identity explained & 62 & 2 & $3.20 \%$ \\
\hline 2 & Preoperative patient status discussed & 62 & 0 & 0 \\
\hline 3 & Type of operation/procedure done explained & 62 & 51 & $82.20 \%$ \\
\hline 4 & Type of anaesthesia explained & 62 & 51 & $82.20 \%$ \\
\hline 5 & Intraoperative vital signs discussed & 62 & 54 & $87.10 \%$ \\
\hline 6 & \multicolumn{2}{|c|}{ Analgesic drugs given during intraoperative period explained 62} & 39 & $62.90 \%$ \\
\hline 7 & Intraoperative fluid management discussed & 62 & 37 & $59.70 \%$ \\
\hline 8 & Intraoperative blood loss and transfusion discussed & 62 & 5 & $8.10 \%$ \\
\hline 9 & Intraoperative clinical incident discussed & 62 & 2 & $3.20 \%$ \\
\hline 10 & Recovery/extubation patient condition explained & 62 & 28 & $45.10 \%$ \\
\hline II & Postoperative order and management briefly discussed & 62 & 2 & $3.20 \%$ \\
\hline
\end{tabular}

\section{Discussion}

Cross-unit patient handover is a crucial process of patient care in the healthcare systems. ${ }^{7}$ The main goal of patient handover is accurate transfer of information about the patient's state to ensure the safety and continuity of patient care. In addition, it is an interactive communication allowing the opportunity for questioning between the patient senders and receivers of patient's information. ${ }^{7}$ Moreover, handover is also one of the most frequent and influential moments of the patient's passage through hospital as it plays a vital role in determining the management plan of the patient. ${ }^{7}$ Anaesthesia is considered as one of the leading specialties in healthcare in terms of securing patient safety. ${ }^{12}$ Postoperative patient handover from operation theatre to recovery room is one of the core aspects patient care provided by anaesthetists. This process can be impacted by time constraints, pressure to work efficiently, effectiveness of communication among 
care providers, up-dating training and availability of standardised postoperative patient handover protocols. ${ }^{13}$ This prospective pre-post intervention audit revealed that the postoperative patient handover practice of graduate anaesthetists at the University of Gondar teaching and referral hospital was substandard following the introduction of the standards in most of the categories. The areas of poor practice were appreciably improved after training except reporting about intraoperative clinical incidents. This finding demonstrated that mere introduction of a standard is inadequate in ensuring the standard is met. We have shown that training is required.

Table 3 Post training results of postoperative patient handover of patients at the recovery room, April 25- May 27, 20I3 (N=62)

\begin{tabular}{|c|c|c|c|c|}
\hline S. No. & Standard & Handover & $\begin{array}{l}\text { Total number of patients for } \\
\text { whom the standards were } \\
\text { addressed }\end{array}$ & $\begin{array}{l}\text { Total } \\
\text { percentage (\%) }\end{array}$ \\
\hline $\mathrm{I}$ & Patient identity explained & 62 & 48 & $77.40 \%$ \\
\hline 2 & Preoperative patient status discussed & 62 & 51 & $82.20 \%$ \\
\hline 3 & Type of operation/procedure done explained & 62 & 62 & $100 \%$ \\
\hline 4 & Type of anaesthesia explained & 62 & 62 & $100 \%$ \\
\hline 5 & Intraoperative vital signs discussed & 62 & 59 & $95.10 \%$ \\
\hline 6 & $\begin{array}{l}\text { Analgesic drugs given during intraoperative period } \\
\text { explained }\end{array}$ & 62 & 57 & $91.90 \%$ \\
\hline 7 & Intraoperative fluid management discussed & 62 & 57 & $91.90 \%$ \\
\hline 8 & Intraoperative blood loss and transfusion discussed & 62 & 49 & $79.00 \%$ \\
\hline 9 & Intraoperative clinical incident discussed & 62 & 28 & $45.10 \%$ \\
\hline 10 & Recovery/extubation patient condition explained & 62 & 59 & $95.10 \%$ \\
\hline II & Postoperative order and management briefly discussed & d 62 & 59 & $95.10 \%$ \\
\hline
\end{tabular}

The first audit revealed that we did not meet any of the set recovery room handover standards (Table 1). The areas of very poor practice included patient identity, preoperative patient condition, intraoperative blood loss, intraoperative incidents, recovery condition and postop management plan. All the above areas were remarkably improved following training except intraoperative clinical incident reporting (anaesthetists are expected to explain about the intraoperative clinical incident whether there was an incident or not to the recovery room staff). Despite this improvement, we only met the targets in $2 / 11$ standards. Moreover, the post-training compliance was still below the target goal, which may be attributed to weaknesses in the training and short-term extinction of acquired skill. Poor postoperative handover from operation room to recovery room staff will increase the risk of postoperative complications for patients, increase stress and workload for recovery room staff. Furthermore, it will also decrease the patient satisfaction and recovery room experience of patients after anaesthesia and surgery. ${ }^{7,8,11}$

\section{Limitations of this audit}

Emergency operations were not included in this audit in which case the problem may be huge. In addition, checklist was not attached with the patients' charts which would potentially improve postoperative patient handover practice.

\section{Conclusion and recommendation}

Although, postoperative patient handover standards were introduced and posted in the operation theatres and recovery room, there was a poor practice in the transfer of information in most of the categories. These areas of practice were remarkably improved following the institution of training except intraoperative clinical incident reporting. Increasing education is a necessary component of improving patient handoffs. However, the post-training compliance is still below the target goal, which may be attributed to weaknesses in the training, short-term extinction of acquired skill, or other reason. Regardless, it seems that the post intervention compliance would have improved further and perhaps met the target goals of compliance if a checklist was attached with the patients' charts during the handoff. We suggest that regular training on postoperative patient handover needs to be provided for both graduate and qualified anaesthetists.
Moreover, regular re-auditing is required until the anaesthetists meet the standards to ensure the continuity of patient safety in the course of postoperative care.

\section{Acknowledgements}

We would like to thank our data collectors.

\section{Conflicts of interest}

The authors declare that there is no conflict of interest.

\section{References}

1. Safe handover. Safe patients. Guidance on clinical handover for clinicians and managers, Australian Medical Association. 2006.

2. Guidance on safe handover. Guidance from the working time directive working party. The Royal College of Surgeons of England. 2007.

3. Safe handover. Safe patients. Guidance on clinical handover for clinicians and managers, British Doctors Committee. 2004.

4. Clinical handover guideline. Sydney South West Area Health Service. 2007.

5. Handover/communication tool guidance for staff. Hampshire community health care. 2010.

6. National Clinical Handover Initiative. Nursing and medical handover in general surgery, emergency medicine and general medicine at the Royal Hobart Hospital. Overarching Standardised Operating Protocol. 2008.

7. Manser T, Foster S. Effective handover communication: An overview of research and improvement efforts. Best Pract Res Clin Anaesthesiol. 2011;25(2):181-191.

8. Kalkman JC. Handover in the perioperative care process. Curr Opin Anesthesiol. 2010;23(6):749-753.

9. The handover of responsibility during an anaesthetic. The Hong Kong College of Anaesthesiologists. 2002.

10. Guideline on the handover of responsibility during an anaesthetic. Australian and New Zealand College of Anaesthetists. 2004.

11. Catchpole RK, Deleval RM, Mcewan A, et al. Patient handover from surgery to intensive care: using formula 1 pit-stop and aviation models to improve safety and quality. Paediatr Anaesth. 2007;17(5):470-478. 
12. Gaba DM. Anaesthesiology as a model for patient safety in health care. BMJ. 2000;320(7237):788-791.
13. Rose M, D Newman S. Factors influencing patient safety during postoperative handover. AANA Journal. 2016;84(5):329-338. 